\title{
Efficient Synthesis and in vitro PDT Effect of Purpurin-18- $N$-Aminoimides
}

\author{
Bing Cun Cui, Min Uk Cha, Jia Zhu Li, Ho Sung Park, II Yoon, and Young Key Shim*
}

\author{
PDT Research Institute, School of Nano System Engineering, Inje University, Gimhae 621-749, Korea \\ *E-mail:ykshim@inje.ac.kr \\ Received May 10, 2010, Accepted September 15, 2010
}

\begin{abstract}
A simple and efficient synthetic route for making purpurin-18- $N$-aminoimides is described. The purpurin-18- $N$ aminoimides are obtained by treatment of purpurin-18 methyl ester with various amines. These new compounds have long wavelength absorptions in the range of $706-711 \mathrm{~nm}$. In preliminary screening, the purpurin- $18-N$-aminoimides have shown promising photosensitizing activity for the cancer cell by in vitro study in photodynamic therapy.
\end{abstract}

Key Words: Purpurin-18 methyl ester, Purpurin-18- $N$-aminoimide, Photodynamic therapy

\section{Introduction}

Photodynamic therapy (PDT) is an experimental cancer treatment modality which selectively destroys cancer cells by interaction of light with a photosensitizing dye, presumably to form singlet oxygen. ${ }^{1-3}$ In continuing efforts to develop candidate photosensitizers for PDT, the design and synthesis of chlorin derivatives having well-defined structure with amphiphilic properties, high selectivity for tumor cell, quick elimination from health cells and strong absorption in the red region of visible spectrum are important challenges in the PDT field. In our lab, we have done a large amount of careful research about the chlorin-based photosensitizers. For example, research on Grampositive bacterial cell $S$. aureus using troponyl methyl (pyro) pheophorbides ${ }^{4}$ and carbohydrate-conjugated chlorin for galectin-3. ${ }^{5}$

DNA-intercalating agents are those antitumor agents that intercalate DNA via chromophores. ${ }^{6}$ DNA intercalating ability and antitumor activity resides in a wide variety of chemical entities. Especially, imide derivative compounds have been reported as a potential class of antitumor agents containing amonafide 1, mitonafide 2, azonafide $3^{7-9}$ (Figure 1) and so on. Encouraged by this result, a group of photosensitizers featuring a chlorin ring system fused to a six-membered cyclic imide structure with a basic $N$-substituent has received considerable attention. Converting the six-membered anhydride ring of purpurin-18 into a six-membered cyclic imide structure was found to greatly increased wavelength absorption and good stability for the requirement of an improved photodynamic therapeutic agent. ${ }^{10-19}$ Although previous report has described the synthesis of purpurin-18-N-(N,N-dimethyl)ethylimide from the chlorin $\mathrm{p}_{6}{ }^{14}$ However, the yield of the purpurinimide formed in this reaction was low. It is therefore important to develop a new process for the synthesis of purpurinimide with a good yield. Also, there is no report regarding its in vitro study.

By varying the substituent on the anhydride ring nitrogen atom, the series of available purpurinimides could be extended. Thus reactions of purpurin-18 with secondary di(tri)amines could give rise of various biological active imide derivatives. In this report we wish to present a general and efficient method for the synthesis of the purpurin-18- $N$-aminoimides from the

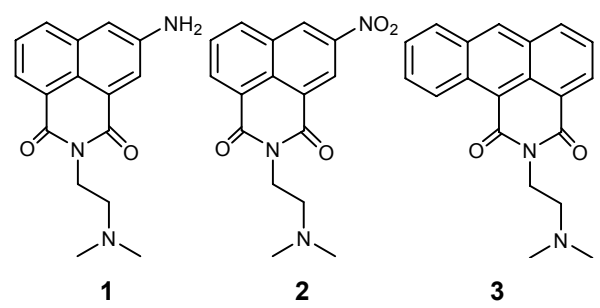

Figure 1. Structures of amonafide 1, mitonafide 2 and azonafide 3.

purpurin-18 methyl ester 5. We also carried out an in vitro assay of cell viability with A549 cancer cells for the aim of examining preliminary photosensitizing efficacy of these new purpurinimides.

\section{Experimental}

General methods. The ${ }^{1} \mathrm{H}-\mathrm{NMR}$ spectra were recorded on a Varian-500 MHz spectrometer. Chemical shifts are given as $\delta$ values using TMS as the internal standard and $J$ values in $\mathrm{Hz}$. The mass spectra were measured with a JEOL JMS700 spectrometer. The UV-visible spectrums were recorded on Scinco S3100 spectrophotometer using dichloromethane as a solvent. Melting points (uncorrected) were measured on an Electrothermal IA9000 Series digital melting point apparatus. Thin-layer chromatography (TLC) was done on Merck silica gel 60 glass sheets (Cat. HX948839, layer thickness $0.25 \mathrm{~mm}$ ). Column chromatography was performed over silica gel 60 (230 - 400 mesh). In some cases, preparative TLC plates were also used for the purification (Analtech precoated silica gel GF glass plate, Cat. 01012, layer thickness $0.5 \mathrm{~mm}$ ). All photophysical experiments were carried out using spectroscopic grade solvents. Purpurin-18 methyl ester ${ }^{12}$ was prepared according to literature procedure.

Methyl pheophorbide-a (4). Chlorophyll paste (excrementum bombycis) (100 g) was dissolved in $500 \mathrm{~mL}$ of $5 \%$ sulfuric acid in methanol and stirred at room temperature for $50 \mathrm{~h}$ under nitrogen atmosphere in dark. Following the standard workup, methyl pheophorbide-a was obtained in $5.1 \%$ yield. The analytical data are identical with those reported previously. ${ }^{20}$ 
General procedure for the preparation of purpurin-18- $\mathrm{N}$ aminoimides. In topical experiment, purpurin-18 methyl ester $5(200 \mathrm{mg})$ and excess of corresponding amine $(0.1 \mathrm{~mL})$ was dissolved in toluene $(20 \mathrm{~mL})$, and the mixture was refluxed under nitrogen atmosphere for $2 \mathrm{~h}$. After monitoring of complete consumption of purpurin-18 methyl ester by TLC, the mixture was cooled to room temperature, solvent and excess amines were removed in vacuum. The crude product was purified by silica column chromatography or preparative TLC plate with an eluent of $10 \%$ methanol in dichloromethane to give corresponding purpurinimide $\mathbf{6 a}, \mathbf{6 b}, \mathbf{6 c}, \mathbf{6 d}$ and $\mathbf{6 e}$ as a purple solid, respectively.

Purpurin-18- $N-(N, N$-dimethyl)ethylimide 6a. Yield: $97 \%$. mp 99 - $101{ }^{\circ} \mathrm{C}$. UV-vis in $\mathrm{CH}_{2} \mathrm{Cl}_{2}, \lambda_{\text {max }}$ (nm, rel. intensity log $\varepsilon$ ), 419.2 (0.98), 481.1 (0.04), 511.9 (0.05), 549.8 (0.17), 649.8 (0.06), 706.7 (0.36). ${ }^{1} \mathrm{H} \mathrm{NMR}\left(500 \mathrm{MHz}, \mathrm{CDCl}_{3}\right) \delta 9.55(\mathrm{~s}, 1 \mathrm{H}$, for $10 \mathrm{H}), 9.32(\mathrm{~s}, 1 \mathrm{H}$, for $5 \mathrm{H}), 8.56(\mathrm{~s}, 1 \mathrm{H}$, for $20 \mathrm{H}), 7.89(\mathrm{dd}$, $\left.J=17.5,11.5 \mathrm{~Hz}, 1 \mathrm{H}, 3^{1} \mathrm{H}\right), 6.30\left(\mathrm{~d}, J=18.0 \mathrm{~Hz}, 1 \mathrm{H}\right.$, trans $\left.-3^{2} \mathrm{H}\right)$, $6.17\left(\mathrm{~d}, J=11.5 \mathrm{~Hz}, 1 \mathrm{H}\right.$, cis- $\left.3^{2} \mathrm{H}\right), 5.33(\mathrm{~m}, 1 \mathrm{H}$ for $17 \mathrm{H}), 4.74(\mathrm{t}$, $\left.J=7.0 \mathrm{~Hz}, 2 \mathrm{H}, \mathrm{N}-\mathrm{CH}_{2}-\mathrm{CH}_{2}-\mathrm{N}-\left(\mathrm{CH}_{3}\right)_{2}\right), 4.34(\mathrm{q}, J=7.5 \mathrm{~Hz}, 1 \mathrm{H}$, $18 \mathrm{H}), 3.78\left(\mathrm{~s}, 3 \mathrm{H}, 12-\mathrm{CH}_{3}\right), 3.61\left(\mathrm{q}, J=8.0 \mathrm{~Hz}, 2 \mathrm{H}, 8^{1} \mathrm{CH}_{2}\right), 3.58$ (s, $\left.3 \mathrm{H}, 17^{2} \mathrm{CO}_{2} \mathrm{CH}_{3}\right), 3.34\left(\mathrm{~s}, 3 \mathrm{H}, 2 \mathrm{CH}_{3}\right), 3.14$ (s, $\left.3 \mathrm{H}, 7 \mathrm{CH}_{3}\right)$, $2.76\left(\mathrm{~s}, 6 \mathrm{H}, \mathrm{N}-\left(\mathrm{CH}_{3}\right)_{2}\right), 2.72-2.68,2.43-2.37$ and $2.05-1.98(\mathrm{~m}$, $6 \mathrm{H}, \mathrm{NCH}_{2}-\mathrm{CH}_{2}-\mathrm{N}-\left(\mathrm{CH}_{3}\right)_{2}, 2 \times 17^{1} \mathrm{H}$ and $\left.2 \times 17^{2} \mathrm{H}\right), 1.77(\mathrm{~d}, J=$ $\left.7.5 \mathrm{~Hz}, 3 \mathrm{H}, 18 \mathrm{CH}_{3}\right), 1.65\left(\mathrm{t}, J=7.5 \mathrm{~Hz}, 3 \mathrm{H}, 8^{2} \mathrm{CH}_{3}\right), 0.08$ and -0.08 (each br s, $1 \mathrm{H}, 2 \mathrm{NH}$ ). Anal. calcd for $\mathrm{C}_{38} \mathrm{H}_{44} \mathrm{~N}_{6} \mathrm{O}_{4}: \mathrm{C}$, 70.35; H, 6.84; N, 12.95. Found: C, 70.47; H, 6.82; N, 12.92.

Purpurin-18- $\mathrm{N}-(\mathrm{N}, \mathrm{N}$-diethyl)ethylimide 6b. Yield: $95 \%$. mp $125-127{ }^{\circ} \mathrm{C}$. UV-vis in $\mathrm{CH}_{2} \mathrm{Cl}_{2}, \lambda_{\max }(\mathrm{nm}$, rel. intensity $\log \varepsilon$ ), 419.4 (1.02), 481.1 (0.04), 512.5 (0.06), 551.1 (0.19), 651.6 (0.08), 708.3 (0.37). ${ }^{1} \mathrm{H}$ NMR $\left(500 \mathrm{MHz}, \mathrm{CDCl}_{3}\right) \delta 9.40(\mathrm{~s}, 1 \mathrm{H}$, for $10 \mathrm{H}), 9.18(\mathrm{~s}, 1 \mathrm{H}$, for $5 \mathrm{H}), 8.55(\mathrm{~s}, 1 \mathrm{H}$, for $20 \mathrm{H}), 7.79(\mathrm{dd}$, $\left.J=17.5,11.5 \mathrm{~Hz}, 1 \mathrm{H}, 3^{1} \mathrm{H}\right), 6.23\left(\mathrm{~d}, J=18.0 \mathrm{~Hz}, 1 \mathrm{H}\right.$, trans $\left.-3^{2} \mathrm{H}\right)$, $6.10\left(\mathrm{~d}, J=11.5 \mathrm{~Hz}, 1 \mathrm{H}\right.$, cis- $\left.3^{2} \mathrm{H}\right), 5.37(\mathrm{~m}, 1 \mathrm{H}$ for $17 \mathrm{H}), 4.59(\mathrm{t}$, $\left.2 \mathrm{H}, J=7.0 \mathrm{~Hz}, \mathrm{~N}-\mathrm{CH}_{2}-\mathrm{CH}_{2}-\mathrm{N}-\left(\mathrm{CH}_{2} \mathrm{CH}_{3}\right)\right), 4.36(\mathrm{q}, J=7.5 \mathrm{~Hz}$, $1 \mathrm{H}, 18 \mathrm{H}), 3.71\left(\mathrm{~s}, 3 \mathrm{H}, 12-\mathrm{CH}_{3}\right), 3.48\left(\mathrm{q}, J=8.0 \mathrm{~Hz}, 2 \mathrm{H}, 8^{1} \mathrm{CH}_{2}\right)$, $3.58\left(\mathrm{~s}, 3 \mathrm{H}, 17^{2} \mathrm{CO}_{2} \mathrm{CH}_{3}\right), 3.30\left(\mathrm{~s}, 3 \mathrm{H}, 2 \mathrm{CH}_{3}\right), 3.09(\mathrm{~s}, 3 \mathrm{H}$, $\left.7 \mathrm{CH}_{3}\right), 2.90$ (q, $\left.4 \mathrm{H}, \mathrm{N}-\left(\mathrm{CH}_{2} \mathrm{CH}_{3}\right)_{2}\right), 2.73-2.68,2.44-2.37$ and 2.10-1.97 (m, 6H, N-CH $-\mathrm{CH}_{2}-\mathrm{N}-\left(\mathrm{CH}_{2} \mathrm{CH}_{3}\right)_{2}, 2 \times 17^{1} \mathrm{H}$ and $2 \times$ $\left.17^{2} \mathrm{H}\right), 1.78\left(\mathrm{~d}, J=7.5 \mathrm{~Hz}, 3 \mathrm{H}, 18 \mathrm{CH}_{3}\right), 1.57(\mathrm{t}, J=7.5 \mathrm{~Hz}, 3 \mathrm{H}$, $\left.8^{2} \mathrm{CH}_{3}\right), 1.27\left(\mathrm{t}, J=7.0 \mathrm{~Hz}, 6 \mathrm{H}, \mathrm{N}-\left(\mathrm{CH}_{2}-\mathrm{CH}_{3}\right)_{2}\right), 0.25$ and -0.02 (each br s, $1 \mathrm{H}, 2 \mathrm{NH}$ ). Anal. calcd for $\mathrm{C}_{40} \mathrm{H}_{48} \mathrm{~N}_{6} \mathrm{O}_{4}$ : C, 70.98; $\mathrm{H}$, 7.15; N, 12.42. Found: C, 70.93; H, 7.20; N, 12.48.

Purpurin-18- $\mathrm{N}-(\mathrm{N}$-isopropylamino $)$ ethylimide $6 \mathrm{c}$. Yield: 94\%. mp $116-118^{\circ} \mathrm{C}$. UV-vis in $\mathrm{CH}_{2} \mathrm{Cl}_{2}, \lambda_{\max }$ (nm, rel. intensity $\log \varepsilon), 419.8$ (0.97), 480.9 (0.05), 512.5 (0.06), 552.6 (0.20), 661.4 (0.09), 710.6 (0.35). ${ }^{1} \mathrm{HNMR}\left(500 \mathrm{MHz}, \mathrm{CDCl}_{3}\right) \delta 9.94(\mathrm{~s}$, $1 \mathrm{H}$, for $10 \mathrm{H}), 8.97(\mathrm{~s}, 1 \mathrm{H}$, for $5 \mathrm{H}), 8.52(\mathrm{~s}, 1 \mathrm{H}$, for $20 \mathrm{H}), 7.80$ $\left(\mathrm{dd}, J=19.5,11.5 \mathrm{~Hz}, 1 \mathrm{H}, 3^{1} \mathrm{H}\right), 6.27(\mathrm{~d}, J=17.5 \mathrm{~Hz}, 1 \mathrm{H}$, trans$\left.3^{2} \mathrm{H}\right), 6.15\left(\mathrm{~d}, J=11.5 \mathrm{~Hz}, 1 \mathrm{H}\right.$, cis- $\left.3^{2} \mathrm{H}\right), 5.27(\mathrm{~m}, 1 \mathrm{H}$ for $17 \mathrm{H})$, 4.84 (m, 2H, N-CH $-\mathrm{CH}_{2}-\mathrm{NH}-$ ), 4.34 (q, $J=7.5 \mathrm{~Hz}, 1 \mathrm{H}, 18 \mathrm{H}$ ), $3.59\left(\mathrm{~s}, 3 \mathrm{H}, 12-\mathrm{CH}_{3}\right), 3.60\left(\mathrm{q}, J=6.5 \mathrm{~Hz}, 2 \mathrm{H}, 8^{1} \mathrm{CH}_{2}\right), 3.57$ (s, $\left.3 \mathrm{H}, 17^{2} \mathrm{CO}_{2} \mathrm{CH}_{3}\right), 3.24\left(\mathrm{~s}, 3 \mathrm{H}, 2 \mathrm{CH}_{3}\right), 2.82\left(\mathrm{~s}, 3 \mathrm{H}, 7 \mathrm{CH}_{3}\right), 2.92$ $\left(\mathrm{m}, 1 \mathrm{H}, \mathrm{NH}-\mathrm{CH}-\left(\mathrm{CH}_{3}\right)_{2}\right), 2.75-2.68,2.43-2.35$ and 2.06-1.91 (m, 6H, N-CH $-\mathrm{CH}_{2}-\mathrm{NH}-, 2 \times 17^{1} \mathrm{H}$ and $\left.2 \times 17^{2} \mathrm{H}\right), 2.04(\mathrm{br}, 1 \mathrm{H}$, $\mathrm{NH}), 1.86\left(\mathrm{~d}, J=8.0 \mathrm{~Hz}, 3 \mathrm{H}, 18 \mathrm{CH}_{3}\right), 1.54\left(\mathrm{t}, J=7.0 \mathrm{~Hz}, 3 \mathrm{H}, 8^{2}-\right.$ $\left.\mathrm{CH}_{3}\right), 1.25\left(\mathrm{~d}, \mathrm{~J}=6.5 \mathrm{~Hz}, 6 \mathrm{H}, \mathrm{NH}-\mathrm{CH}-\left(\mathrm{CH}_{3}\right)_{2}\right), 0.09$ and -0.62 (each br s, $1 \mathrm{H}, 2 \mathrm{NH}$ ). Anal. calcd for $\mathrm{C}_{39} \mathrm{H}_{46} \mathrm{~N}_{6} \mathrm{O}_{4}$ : C, 70.67; $\mathrm{H}$,
7.00; N, 12.68. Found: C, 70.69; H, 7.06; N, 12.61.

Purpurin-18- $\mathbf{N}-(\mathrm{N}, \mathrm{N}$-dimethylpropylamino)propylimide 6d. Yield: $97 \%$. mp $95-97{ }^{\circ} \mathrm{C}$. UV-vis in $\mathrm{CH}_{2} \mathrm{Cl}_{2}, \lambda_{\max }$ (nm, rel. intensity $\log \varepsilon$ ), 419.1 (0.98), 481.0 (0.04), 511.7 (0.06), 550.0 (0.17), 651.3 (0.07), 706.9 (0.35). ${ }^{1} \mathrm{H} \mathrm{NMR}\left(500 \mathrm{MHz}, \mathrm{CDCl}_{3}\right)$ $\delta 9.52(\mathrm{~s}, 1 \mathrm{H}$, for $10 \mathrm{H}), 9.29(\mathrm{~s}, 1 \mathrm{H}$, for $5 \mathrm{H}), 8.55(\mathrm{~s}, 1 \mathrm{H}$, for $20 \mathrm{H}), 7.86\left(\mathrm{dd}, J=17.5,13.5 \mathrm{~Hz}, 1 \mathrm{H}, 3{ }^{1} \mathrm{H}\right), 6.28(\mathrm{~d}, J=18.0 \mathrm{~Hz}$, $1 \mathrm{H}$, trans $\left.-3^{2} \mathrm{H}\right), 6.15\left(\mathrm{~d}, J=11.5 \mathrm{~Hz}, 1 \mathrm{H}\right.$, cis- $\left.3^{2} \mathrm{H}\right), 5.31(\mathrm{~m}, 1 \mathrm{H}$ for $17 \mathrm{H}), 4.56$ (m, $\left.2 \mathrm{H}, \mathrm{N}-\mathrm{CH}_{2}-\mathrm{CH}_{2}-\mathrm{CH} 2-\mathrm{NH}-\right), 4.35$ (q, $J=7.0$ $\mathrm{Hz}, 1 \mathrm{H}, 18 \mathrm{H}), 3.75$ (s, 3H, 12- $\left.\mathrm{CH}_{3}\right), 3.57$ (q, $J=8.5 \mathrm{~Hz}, 2 \mathrm{H}$, $\left.8^{1} \mathrm{CH}_{2}\right), 3.55\left(\mathrm{~s}, 3 \mathrm{H}, 17^{2} \mathrm{CO}_{2} \mathrm{CH}_{3}\right), 3.33\left(\mathrm{~s}, 3 \mathrm{H}, 2 \mathrm{CH}_{3}\right), 3.10(\mathrm{~s}$, $\left.3 \mathrm{H}, 7 \mathrm{CH}_{3}\right), 2.70-2.62,2.41-2.10\left(\mathrm{~m}, 8 \mathrm{H}, \mathrm{N}-\mathrm{CH}_{2}-\mathrm{CH}_{2}-\mathrm{CH}_{2}-\mathrm{NH}-\right.$ $\mathrm{CH}_{2}-\mathrm{CH}_{2}-\mathrm{CH}_{2}-\mathrm{N}-\left(\mathrm{CH}_{3}\right), 2 \times 17^{1} \mathrm{H}$ and $\left.2 \times 17^{2} \mathrm{H}\right), 2.05(\mathrm{~s}, 6 \mathrm{H}$, $\left.\mathrm{N}-\left(\mathrm{CH}_{3}\right)_{2}\right) 1.76\left(\mathrm{~d}, J=7.5 \mathrm{~Hz}, 3 \mathrm{H}, 18 \mathrm{CH}_{3}\right), 1.63(\mathrm{t}, J=7.0 \mathrm{~Hz}$, $\left.3 \mathrm{H}, 8^{2} \mathrm{CH}_{3}\right), 1.36\left(\mathrm{~m}, 2 \mathrm{H}, \mathrm{N}-\mathrm{CH}_{2}-\mathrm{CH}_{2}-\mathrm{CH}_{2}-\mathrm{NH}-\mathrm{CH}_{2}-\mathrm{CH}_{2}-\mathrm{CH}_{2}-\right.$ $\left.\mathrm{N}-\left(\mathrm{CH}_{3}\right)\right), 0.08$ and -0.01 (each br s, $1 \mathrm{H}, 2 \mathrm{NH}$ ). Anal. calcd for $\mathrm{C}_{42} \mathrm{H}_{53} \mathrm{~N}_{7} \mathrm{O}_{4}$ : C, 70.07; H, 7.42; N, 13.62. Found: C, 70.02; H, $7.49 ; \mathrm{N}, 13.60$.

Purpurin-18- $\mathrm{N}-(\mathrm{N}$-imidazolyl)propylimide $6 \mathrm{e}$. Yield: $96 \%$. mp $86-88^{\circ} \mathrm{C}$. UV-vis in $\mathrm{CH}_{2} \mathrm{Cl}_{2}, \lambda_{\max }(\mathrm{nm}$, rel. intensity $\log \varepsilon$ ), 418.9 (1.04), 480.8 (0.05), 511.9 (0.06), 550.4 (0.18), 651.7 (0.07), 707.6 (0.34). ${ }^{1} \mathrm{H} \mathrm{NMR}\left(500 \mathrm{MHz}, \mathrm{CDCl}_{3}\right) \delta 9.53(\mathrm{~s}, 1 \mathrm{H}$, for $10 \mathrm{H}), 9.31(\mathrm{~s}, 1 \mathrm{H}$, for $5 \mathrm{H}), 8.56(\mathrm{~s}, 1 \mathrm{H}$, for $20 \mathrm{H}), 7.88(\mathrm{dd}$, $\left.J=18.0,11.5 \mathrm{~Hz}, 1 \mathrm{H}, 3{ }^{1} \mathrm{H}\right), 7.15,7.08$ (s, 3H, imidazole-H), 6.29 $\left(\mathrm{d}, J=17.5 \mathrm{~Hz}, 1 \mathrm{H}\right.$, trans $\left.-3^{2} \mathrm{H}\right), 6.16(\mathrm{~d}, J=11.5 \mathrm{~Hz}, 1 \mathrm{H}$, cis$\left.3^{2} \mathrm{H}\right), 5.33(\mathrm{~m}, 1 \mathrm{H}$ for $17 \mathrm{H}), 4.35$ (q, $\left.J=7.5 \mathrm{~Hz}, 1 \mathrm{H}, 18 \mathrm{H}\right), 4.55$, 4.28 (each m, $2 \mathrm{H}, \mathrm{N}-\mathrm{CH}_{2}-\mathrm{CH}_{2}-\mathrm{CH}_{2}-$ ), 3.77 (s, 3H, 12- $\mathrm{CH}_{3}$ ), 3.59 $\left(\mathrm{q}, J=8.0 \mathrm{~Hz}, 2 \mathrm{H}, 8^{1} \mathrm{CH}_{2}\right), 3.53\left(\mathrm{~s}, 3 \mathrm{H}, 17^{2} \mathrm{CO}_{2} \mathrm{CH}_{3}\right), 3.34(\mathrm{~s}, 3 \mathrm{H}$, $\left.2 \mathrm{CH}_{3}\right), 3.13\left(\mathrm{~s}, 3 \mathrm{H}, 7 \mathrm{CH}_{3}\right), 2.78-2.65,2.55-2.48,2.45-2.32$ and 2.06-1.95(m, 6H, N-CH $-\mathrm{CH}_{2}-\mathrm{CH}_{2-}, 2 \times 17^{1} \mathrm{H}$ and $\left.2 \times 17^{2} \mathrm{H}\right)$, $1.77\left(\mathrm{~d}, J=7.5 \mathrm{~Hz}, 3 \mathrm{H}, 18 \mathrm{CH}_{3}\right), 1.64\left(\mathrm{t}, J=7.8 \mathrm{~Hz}, 3 \mathrm{H}, 8^{2} \mathrm{CH}_{3}\right)$, 0.88 and 0.03 (each br s, $1 \mathrm{H}, 2 \mathrm{NH}$ ). Anal. calcd for $\mathrm{C}_{40} \mathrm{H}_{43} \mathrm{~N}_{7} \mathrm{O}_{4}$ : C, 70.05; H, 6.32; N, 14.30. Found: C, 70.13; H, 6.37; N, 14.26.

\section{In vitro photosensitizing efficacy.}

General method: A549 cells were cultured at $37^{\circ} \mathrm{C}$ in a humidified 5\% $\mathrm{CO}_{2}$ incubator using RFMI 1640 growth medium supplemented with $10 \%$ fetal bovine serum and $1 \%$ penicillin/ streptomycin. For phototoxicity studies, A549 cells were plated in 96-well plates at a density of $10 \times 10^{4}$ cells well. After $24 \mathrm{~h}$ of incubation, $100 \mu \mathrm{L}$ of $1 \mu \mathrm{M}, 2 \mu \mathrm{M}, 5 \mu \mathrm{M}, 10 \mu \mathrm{M}$ and $15 \mu \mathrm{M}$ purpurin-18- $N$-aminoimides were added, respectively. Plates were returned to the incubator for $24 \mathrm{~h}$. And then the cells were replaced with fresh media and exposed to light (BioSpec LED, $670-700 \mathrm{~nm}, 2.0 \mathrm{~J} / \mathrm{cm}^{2}$ ) for $15 \mathrm{~min}$. Following illumination, the plates were incubated at $37^{\circ} \mathrm{C}$ in the dark. Every 3, 24, 48 hours later, WST-1 was put into each well and measure the absorbance on $450 \mathrm{~nm}$ wavelength after photoirradiation or without light, respectively. Each experiment was done with three replicate wells. The percentage cell survival was calculated by normalization with respect to the value for no photosensitizer treatment.

\section{Results and Discussion}

For our study, methyl pheophorbide-a (MPa) 4 was extracted from chlorophyll paste (excrementum bombycis) by slight modifications of the previously reported procedure in $5.1 \%$ yield. ${ }^{20}$ $\mathrm{MPa} 4$ was transformed into purpurin-18 methyl ester $\mathbf{5}^{6}$ by 


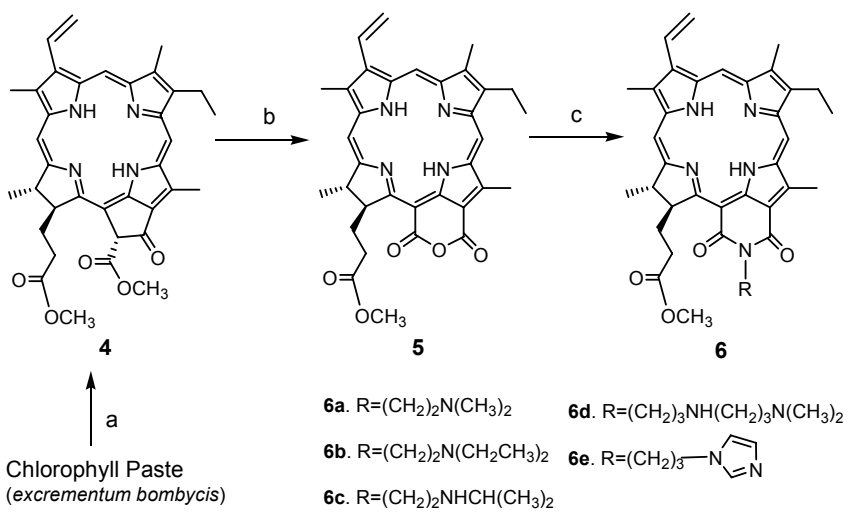

Scheme 1. Synthesis of purpurin-18- $N$-aminoimides. Reagents and conditions: a. 5\% $\mathrm{H}_{2} \mathrm{SO}_{4} / \mathrm{Methanol}$, rt; b. $\mathrm{KOH} / 1$-propanol/air, rt; c. Corresponding amines/toluene, reflux

air oxidation in $n$-propanol containing $\mathrm{KOH}$ and was used as a substrate. As shown in Scheme 1, reaction of 5 with various amines with reflux gave the desired purpurin-18-aminoimides $\mathbf{6 a}, \mathbf{6 b}, \mathbf{6 c}, \mathbf{6 d}$ and $\mathbf{6 e}$ in $97 \%, 95 \%, 94 \%, 97 \%$ and $96 \%$ yield, respectively. Thus, this method appeared to be an efficient approach for preparing photosensitizers with $N$-amino-imide substituents that can be prepared in excellent yield directly from purpurin-18 methyl ester via one-pot synthesis.

The structures of the final products were confirmed by ${ }^{1} \mathrm{H}$ NMR spectroscopy and mass spectrometry. Compared to the purpurin-18 methyl ester 5 the ${ }^{1} \mathrm{H}-\mathrm{NMR}$ spectra of $\mathbf{6 a - 6 e}$ showed each triplet at $\delta 4.74,4.59,4.84,4.56$ and $4.55 \mathrm{ppm}$ for the protons of CO-N- $\mathrm{CH}_{2-}$, respectively. In compounds $\mathbf{6 a}$ and $\mathbf{6 d}$, $\mathrm{N}-\left(\mathrm{CH}_{3}\right)_{2}$ appears as a singlet at $\delta 2.76$ and $2.05 \mathrm{ppm}$, respectively. And compound $\mathbf{6 b}$ shows a triplet at $\delta 1.27 \mathrm{ppm}$ for the protons of $\mathrm{N}-\left(\mathrm{CH}_{2} \mathrm{CH}_{3}\right)_{2}$. Compound $\mathbf{6} \mathbf{c}$ showed the appearance of a doublet at $\delta 1.25$ ppm assigned to $\mathrm{NH}-\mathrm{CH}-\left(\mathrm{CH}_{3}\right)_{2}$ protons. The imidazole protons of compound $\mathbf{6 e}$ gave two singlet at $\delta 7.15$ and $7.08 \mathrm{ppm}$.

The electronic absorption spectra of purpurin-18- $N$-aminoimides were compared with purpurin-18 methyl ester. All compounds showed the same pyrrole-type visible spectra in dichloromethane, but the peaks in max of Qy and Soret bands are different. As can be seen from Figure 2, all the chlorins showed similar characteristics. The Soret band was observed near 419 $\mathrm{nm}$, and the long-wavelength absorption bands were observed at $\lambda_{\max }=706.7,708.3,710.6,706.9$ and $707.6 \mathrm{~nm}$ for $\mathbf{6 a}, \mathbf{6 b}$, $\mathbf{6 c}, \mathbf{6 d}$ and $\mathbf{6 e}$, respectively. Thus comparing with purpurin-18 methyl ester $\mathbf{5}\left(\lambda_{\max }=699.9 \mathrm{~nm}\right)$, the purpurin-18-aminoimides $\mathbf{6 a}, \mathbf{6 b}, \mathbf{6 c}, \mathbf{6 d}$ and $\mathbf{6 e}$, showed a red-shift of 6.8, 8.4, 10.7, 7.0, $7.7 \mathrm{~nm}$, respectively (Table 1). In other words the use of photosensitizer having long-wavelength band is desirable for better tissue penetration of excitied light in vivo, the purpurin-18- $\mathrm{N}$ aminoimides may be considered as promising candidates. ${ }^{19,21}$

The in vitro activities of purpurin-18 methyl ester 5 and the corresponding purpurin-18- $N$-aminoimides were determined with A549 cells. A549 cells with each compound at variable concentrations were incubated in A549 cells at $37{ }^{\circ} \mathrm{C}$ for $3 \mathrm{~h}$, $24 \mathrm{~h}$ and $48 \mathrm{~h}$ respectively. As shown in Figure 3, none of the

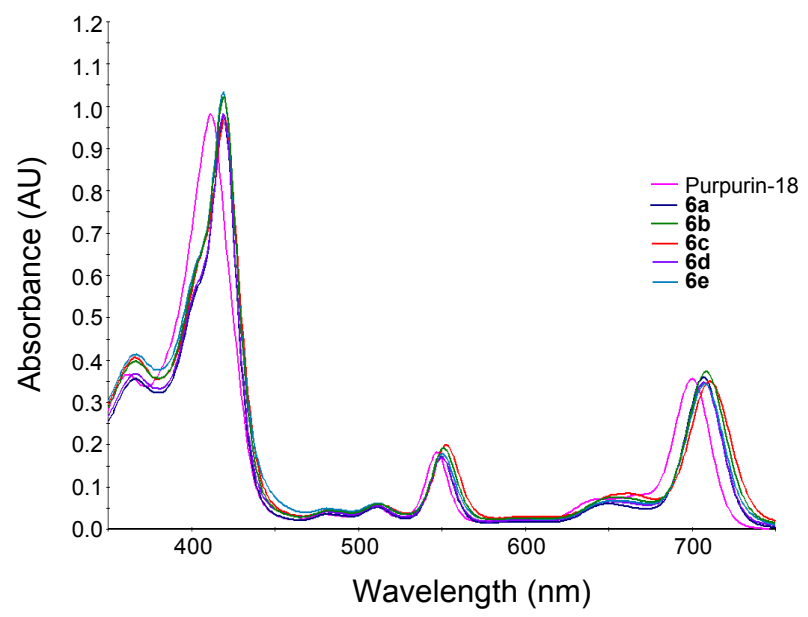

Figure 2. Electronic absorption spectra of 6a-6e in dichloromethane.

Table 1. Absorption maxima of $6 \mathrm{a}-6 \mathrm{e}$ in dichloromethane and their redshift values of the redmost Qy band

\begin{tabular}{cccc}
\hline \multirow{2}{*}{ compound } & \multicolumn{2}{c}{ Absorption maxima, $\mathrm{nm}$} & \multirow{2}{*}{$\Delta \mathrm{Qy}(\mathrm{nm})$} \\
\cline { 2 - 3 } & Soret & Redmost Qy & \\
\hline 6 & 411.4 & 699.9 & 0 \\
6a & 419.2 & 706.7 & 6.8 \\
6b & 419.4 & 708.3 & 8.4 \\
6c & 419.8 & 710.6 & 10.7 \\
6d & 419.1 & 706.9 & 7.0 \\
6e & 418.9 & 707.6 & 7.7 \\
\hline
\end{tabular}

compounds up to $15 \mu \mathrm{M}$ concentration have any significant dark cytotoxicity at $3 \mathrm{~h}$ and $24 \mathrm{~h}$. But the compounds showed little dark cytotoxicity at $48 \mathrm{~h}$, presumably by light penetration errors during long time incubation. The higher concentration of the photosensitizer the more dark cytotoxicity was shown. Among the compounds investigated, compound $\mathbf{6 b}$ showed the best efficacy at $3 \mathrm{~h}$ with $16.1 \%$ cell survival at $2 \mu \mathrm{M}$. After $24 \mathrm{~h}$ incubation in the dark, all the purpurin-18- $N$-aminoimides exhibited similar PDT efficacy. However, compared to the standard purpurin-18 methyl ester 5, all the purpurin-18-N-aminoimides showed higher PDT efficacy. The in vitro efficacy after $24 \mathrm{~h}$ incubation was observed in the following order: $\mathbf{6 c}>\mathbf{6 e}>\mathbf{6 a}>$ $\mathbf{6 d}>\mathbf{6 b}>\mathbf{5}$. And the dark cytotoxicity was 3\%, 5\%, 3\%, 3\% and $5 \%$ for compounds $6 \mathbf{6}, \mathbf{6 b}, \mathbf{6 c}, \mathbf{6 d}$ and $\mathbf{6 e}$ at $2 \mu \mathrm{M}$ after $24 \mathrm{~h}$, respectively.

\section{Conclusions}

We have developed a simple and efficient synthetic route for preparing the purpurin $18-\mathrm{N}$-aminoimides. Reaction of 5 with various amines at reflux gave the corresponding imides in excellent yield ( $>94 \%)$. On the basis of the preliminary in vitro studies, it is clearly indicated that the purpurin-18- $N$-aminoimides exhibit better PDT efficacy than the standard purpurin-18 methyl ester $\mathbf{5}$. 
PDT, $3 \mathrm{~h}$

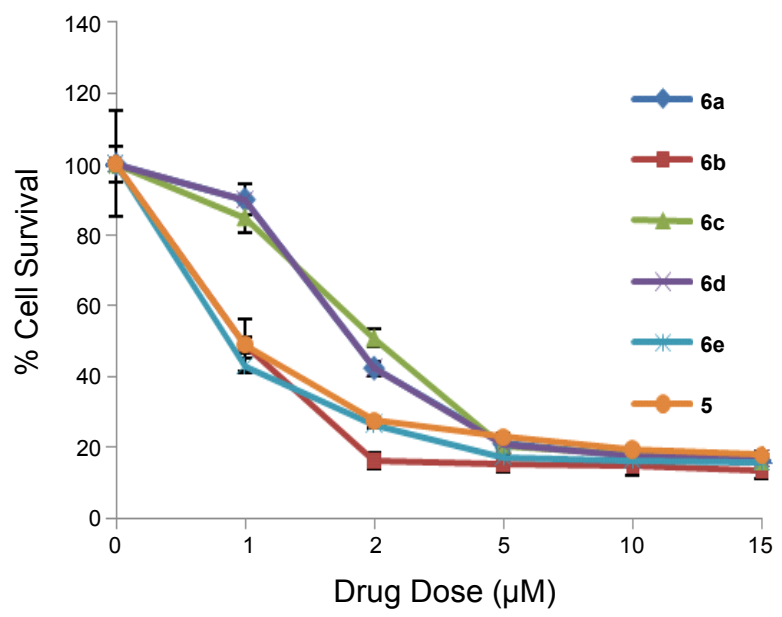

PDT, $24 \mathrm{~h}$

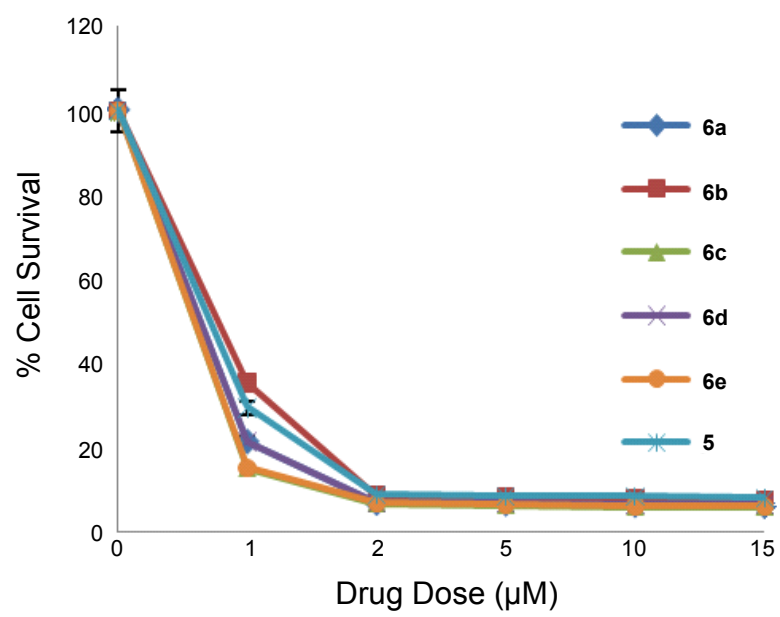

PDT, $48 \mathrm{~h}$

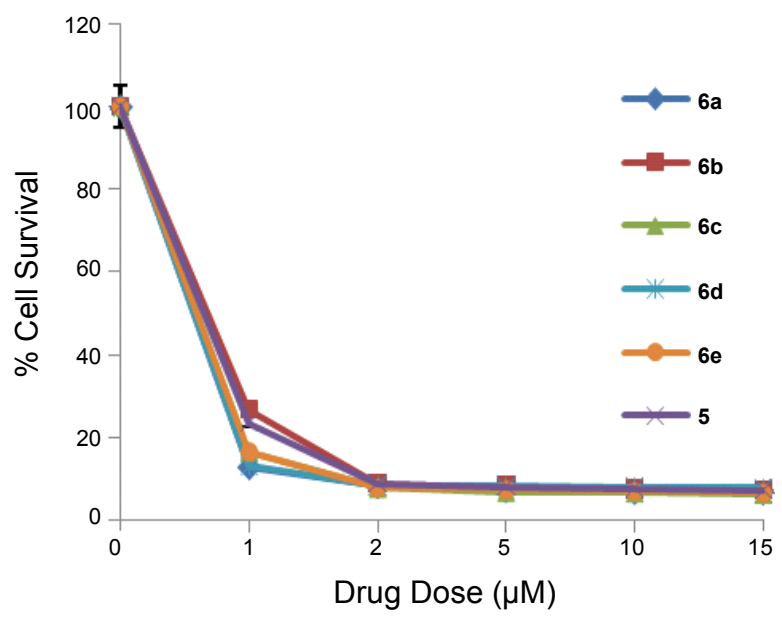

Dark, $3 \mathrm{~h}$

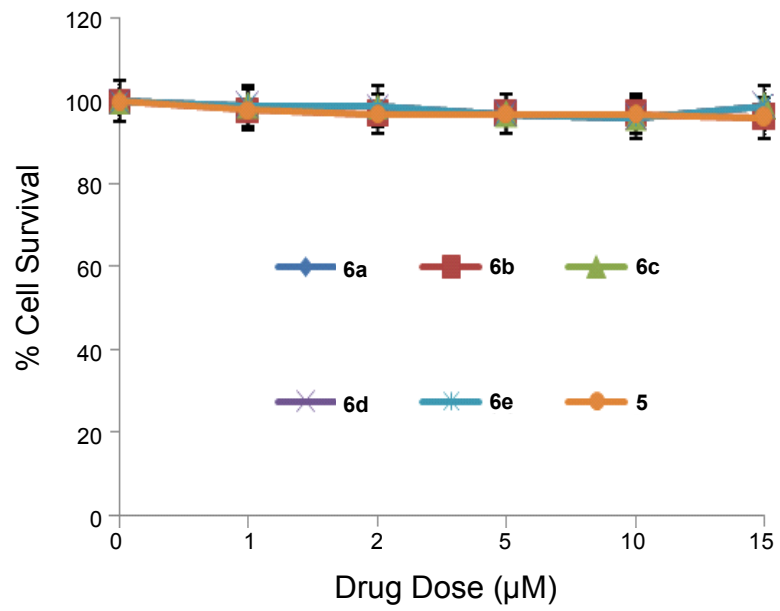

Dark, $24 \mathrm{~h}$

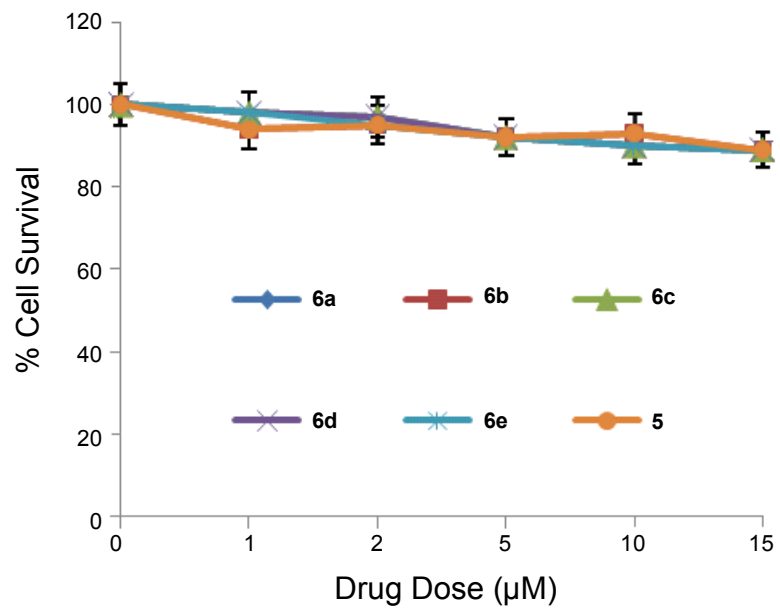

Dark, $48 \mathrm{~h}$

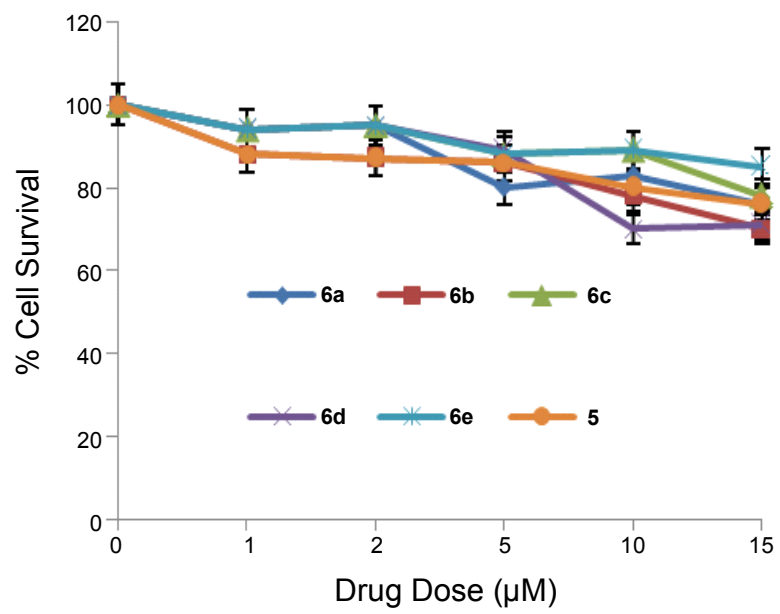

Figure 3. Cell viability results of the photosensitizers on A549 cells. The cell viability of 6a-6e was assigned for PDT in the concentration ranges of $1 \mu \mathrm{M}, 2 \mu \mathrm{M}, 5 \mu \mathrm{M}, 10 \mu \mathrm{M}$ and $15 \mu \mathrm{M}$ after $3 \mathrm{~h}, 24 \mathrm{~h}$, and $48 \mathrm{~h}$ by comparing with those of purpurin-18 methyl ester. 
Acknowledgments. This work was supported by Inje-University Research Grant in 2010.

\section{References}

1. MacDonald, I. J.; Dougherty, T. J. J. Porphyrins Phthalocyanines 2001, 5, 105 .

2. Gomer, C. J.; Rucker, N.; Ferrario, A.; Wong, S. Radiat. Res. 1989, 120,1 .

3. Nyman, E. S.; Hynninen, P. H. J. Photochem. Photobiol. B 2004, 73,1 .

4. Bold, B.; Barkhuu, B.; Lee, W. K.; Shim, Y. K. Bull. Korean Chem. Soc. 2008, 29, 237.

5. Park, Y. K.; Bold, B.; Cui, B. C.; Bai, J. Q.; Lee, W. K.; Shim, Y. K. Bull. Korean Chem. Soc. 2008, 29, 130.

6. Brana, M. F.; Cacho, M.; Gradillas, A.; Pascual-Teresa, B.; Ramos, A.; Curr. Pharm. Res. 2001, 7, 1745.

7. Sami, S. M.; Dorr, R. T.; Alberts, D. S.; Remers, W. A. J. Med. Chem. 1993, 6, 765.

8. Brana, M. F.; Castellano, J. M.; Moran, M.; Emling, F.; Kluge, M.; Schlick, E.; Klebe, G.; Walker, N. Arzneimittelforschung. 1995, 45, 1311.

9. Sami, S. M.; Dorr, R. T.; Alberts, D. S.; Solyom, A. M.; Remers, W. A. J. Med. Chem. 1996, 39, 4978.

10. Karuso, P.; Bergquist, P. R.; Buckleton, I. S.; Cambie, R. C.; Clar,
G. R.; Richard, C. E. F. Tetrahedron Lett. 1986, 27, 2177.

11. Dolmans, E. J. G. J.; Kadami, A.; Hill, S. J.; Waters, C. A.; Robinson, B, C.; Walker, J. P.; Fukumura, D.; Jain, R. K. Cancer Res. 2002, 62, 2151.

12. Zheng, G.; Potter, R. P.; Camacho, S. H.; Missert, R.; Wang, G. S.; Belliner, D. A.; Hendersom, B. W.; Rodgers, M. A. J.; Dougherty, T. J.; Pandey, R. K. J. Med. Chem. 2001, 44, 1540.

13. Lee, S. H.; Jagerovic, N.; Smith, K. V. J. Chem. Soc. Perkin trans 1 1993, 19, 2369.

14. Kozyrev. A. N.; Zheng, G.; Lazarou, E.; Dougherty, T. J.; Smith, K. M.; Pandey, R. K. Tetrahedron Lett. 1997, 19, 3335.

15. Rungta, A.; Zheng, G.; Missert, J. R.; Potter, W. R.; Dougherty, T. J.; Pandey, R. K. Bioorg. Med. Chem. Lett. 2000, 10, 1463.

16. Mironov, A. F.; Lebedeva, V. S. Tetrahedron Lett. 1998, $39,905$.

17. Mironov, A. F.; Grin, M. A.; Tsyprovskiy, A. G. J. Porphyrins Phthalocyanines 2002, 6, 358 .

18. Kozyrev. A. N.; Zheng, G.; Zhu, C. F.; Dougherty, T. J.; Smith, K. M.; Pandey, R. K. Tetrahedron Lett. 1996, 37, 6431.

19. Chen, Y.; Graham, A.; Potter, W.; Morgan, J.; Vaughan, L.; Bellnier, D. A.; Henderson, B. W.; Oseroff, A.; Dougherty, T. J.; Pandey, R. K. J. Med. Chem. 2002, 45, 255.

20. Smith, K. M.; Goff, D. A.; Simpson, D. J. J. Am. Chem. Soc. 1985, 107, 4946.

21. Zheng, G.; Potter, W. R.; Sumlin, A. B.; Dougherty, T. J.; Pandey, R. K. Bioorg. Med. Chem. Lett. 2000, 10, 123. 